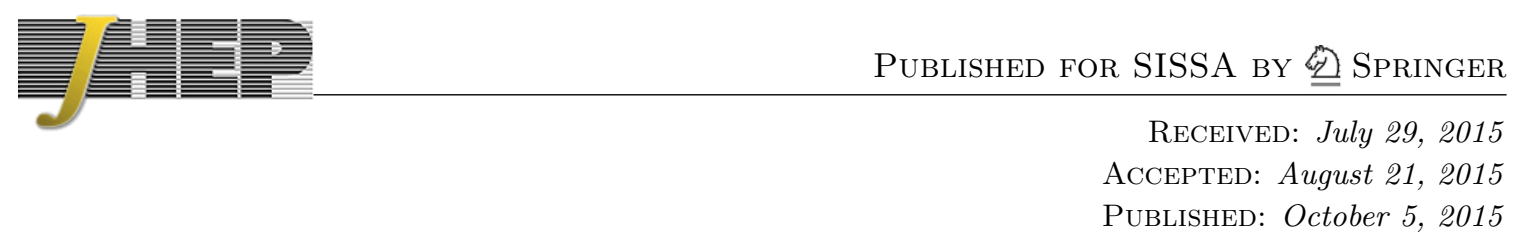

\title{
Instantons in Lifshitz field theories
}

\section{Toshiaki Fujimori and Muneto Nitta}

Department of Physics, and Research and Education Center for Natural Sciences, Keio University, Hiyoshi 4-1-1, Yokohama, Kanagawa 223-8521, Japan

E-mail: toshiaki.fujimori018@gmail.com, nitta@phys-h.keio.ac.jp

ABSTRACT: BPS instantons are discussed in Lifshitz-type anisotropic field theories. We consider generalizations of the sigma model/Yang-Mills instantons in renormalizable higher dimensional models with the classical Lifshitz scaling invariance. In each model, BPS instanton equation takes the form of the gradient flow equations for "the superpotential" defining "the detailed balance condition". The anisotropic Weyl rescaling and the coset space dimensional reduction are used to map rotationally symmetric instantons to vortices in two-dimensional anisotropic systems on the hyperbolic plane. As examples, we study anisotropic BPS baby Skyrmion 1+1 dimensions and BPS Skyrmion in $2+1$ dimensions, for which we take Kähler 1-form and the Wess-Zumiono-Witten term as the superpotentials, respectively, and an anisotropic generalized Yang-Mills instanton in $4+1$ dimensions, for which we take the Chern-Simons term as the superpotential.

Keywords: Solitons Monopoles and Instantons, Space-Time Symmetries, Sigma Models, Nonperturbative Effects

ArXiv EPrint: 1507.06456 


\section{Contents}

1 Introduction 1

2 Instantons in Lifshitz-type sigma models 3

2.1 Derrick's scaling argument for Lifshitz-type sigma models 3

2.2 "Supersymmetric" models and BPS equations 4

2.3 Anisotropic BPS baby Skyrmions $\quad 6$

$\begin{array}{lll}2.4 & \text { Anisotropic BPS Skyrmions } & 7\end{array}$

2.4.1 $G=\mathrm{SU}(2) \quad 8$

$\begin{array}{lll}2.5 & \text { Instantons in higher dimensional sigma models } & 12\end{array}$

$\begin{array}{lll}2.5 .1 & O(d+1) \text { sigma model } & 12\end{array}$

$\begin{array}{lll}2.5 .2 & \text { Other examples } & 13\end{array}$

3 Instantons in Lifshitz-type gauge theories $\quad 14$

3.1 Derrick's scaling argument in Lifshitz-type gauge theories 14

$\begin{array}{lll}3.2 & \text { Weyl rescaling and coset space dimensional reduction } & 15\end{array}$

$\begin{array}{lll}3.3 & \text { Generalized Yang-Mills instantons } & 15\end{array}$

$\begin{array}{lll}3.3 .1 & (5+1) \text {-dimensional SU(4) gauge theory } & 16\end{array}$

4 Summary and discussion $\quad 20$

$\begin{array}{ll}\text { A A supersymmetry in Lifshitz-type theories } & 21\end{array}$

\section{Introduction}

Instantons are important objects which are relevant to non-perturbative phenomena in various physical system. They are characterized as topologically non-trivial field configurations which are stationary points of the Euclidean action of the system, and hence they give non-perturbative contributions to path integrals. Well-known examples of instantons include the sigma model instantons in two dimensional non-linear sigma models and the Yang-Mills instantons in four dimensional non-Abelian gauge theories. Both models are renormalizable asymptotically free quantum field theories which have rich non-perturbative structures in the low-energy regime.

Although the straightforward generalizations of those theories to higher dimensions are known to be perturbatively non-renormalizable, there are renormalizable higher dimensional generalizations of the sigma model and the gauge theory. Such theories, called the Lifshitz-type fields theories, are characterized by the following anisotropic scaling of the spacetime coordinates $[1,2]$

$$
t \rightarrow \lambda^{z} t, \quad x_{i} \rightarrow \lambda x_{i}
$$


This scaling transformation is called the Lifshitz scaling with the dynamical critical exponent $z$. Because of the anisotropy between space and time, the Lorentz symmetry is explicitly broken, whereas the spatial rotational symmetry is preserved. The spatial higher derivative terms in the Lagrangian improve the ultraviolet (UV) behaviors of the propagators so that the UV divergences of loop integrals become milder than those in the standard Lorentz symmetric theories. The absence of higher time derivative terms guarantees that the Lifshitz-type theories are free from the ghost problem associated with higher derivatives.

Using the idea of the Lifshitz scaling, Horava constructed a gravity theory [3, 4], called "the Horava-Lifshitz gravity", which is expected to be renormalizable and unitary. In $(d+1)$ dimensional spacetime, non-linear sigma models with $z=d$ and Yang-Mills theories with $z=d-2$ are classically invariant under the Lifshitz scaling transformation, and expected to be renormalizable according to the weighted power counting [5-7] and a symmetry argument [8]. The various aspects of the Lifshitz-type theories have been discussed in the non-linear sigma models [9-12] and in the Yang-Mills theories [13-15] (see [16] for a review). In most of those cases, the theories are asymptotically free (or scale invariant) and it is likely that their strongly coupled low-energy dynamics have rich non-perturbative structures induced by instantons.

In recent years, BPS solitons has been extensively studied in a certain class of Lorentz symmetric non-linear sigma models with higher derivative terms [17-33]. Those models are invariant under the volume-preserving diffeomorphism, which allows one to determine various aspects of solitons. It is also possible to implement such an infinite dimensional symmetry in the Lifshitz-type non-linear sigma models as the spatial volume-preserving diffeomorphism. BPS solitons in such Lifshitz-type models were discussed in [34] (another kind of BPS solitons in Lifshitz field theories have also been discussed in [35]).

In this paper, we discuss instantons in classically scale invariant Lifshitz-type nonlinear sigma models and gauge theories in the Wick-rotated spacetime. We focus on "supersymmetric theories" which satisfy "the detailed balance condition" characterized by a functional version of the superpotential. Such models admit BPS instantons described by the gradient flow equations for the superpotential (similar geometric flow equations have been discussed as instantons in the Horava-Lifshitz gravity [36, 37]). A class of non-linear sigma models which is invariant under the Lifshitz scaling and the spatial volume-preserving diffeomorphism can be constructed by adopting closed forms on the target space as the superpotential. As examples we study an anisotropic baby Skyrmion in $1+1$ dimensions and BPS Skyrmion in 2+1 dimensions, for which we take the Kähler 1-form and the WessZumino-Witten term [38] as the superpotentials, respectively. For gauge theories, we use the Chern-Simons form to construct scale invariant models. In both cases, we consider rotationally invariant instantons and reduce the system to the two-dimensional half-plane endowed with the hyperbolic metric.

The organization of this paper is as follows. In section 2, we discuss BPS instantons in the Lifshitz-type non-linear sigma model. After generalizing Derrick's scaling argument [39] to models without the Lorentz symmetry in section 2.1, we determine the Lifshitz-type sigma model action which admits BPS instantons in section 2.2. As examples, we consider 
anisotropic BPS (baby) Skyrmions in sections 2.3 and 2.4. In section 2.5, generalizations to higher dimensional sigma models are discussed. Section 3 is devoted to the instantons in the Lifshitz-type gauge theories. In section 3.1, we generalize the scaling argument to the Lifshitz-type gauge theories. In section 3.2, the reduction of the system by using the anisotropic Weyl rescaling and the coset space dimensional reduction are discussed. An explicit example of the generalized Yang-Mills instanton in (5+1)-dimensional SU(4) gauge theory is discussed in section 3.3. Appendix A is devoted to the superfield formalism for the $(2+1)$-dimensional Lifsthiz-type supersymmetric non-linear sigma model.

\section{Instantons in Lifshitz-type sigma models}

In this section, we consider instantons in the Lifshitz-type non-linear sigma models. The degrees of freedom is a map from the $(d+1)$-dimensional spacetime to a target manifold $\mathcal{M}$ and the scalar fields $\phi^{a}$ are identified with the coordinates of $\mathcal{M}$. The generic form of the Euclidean action is

$$
S=\int d t d^{d} x\left[g_{a b} \partial_{t} \phi^{a} \partial_{t} \phi^{b}+V\left(\phi, \partial_{i} \phi, \cdots\right)\right],
$$

where $g_{a b}$ is the metric on $\mathcal{M}$ and $V\left(\phi, \partial_{i} \phi, \cdots\right)$ is a function of the fields $\phi^{a}$ and their spatial derivatives $\partial_{i} \phi^{a}, \partial_{i} \partial_{j} \phi^{a}$, etc. The model of this type is renormalizable if $V\left(\phi, \partial_{i} \phi, \cdots\right)$ contains a sufficient number of spatial derivatives so that the propagator decreases sufficiently quickly in the UV regime. In the following, we discuss instantons in such models with higher spatial derivatives.

\subsection{Derrick's scaling argument for Lifshitz-type sigma models}

First, we discuss the stability of instantons in the Lifshitz-type theories by using Derrick's scaling argument [39]. Let us consider the following scaling transformation

$$
\phi^{a}\left(x_{i}\right) \rightarrow \phi^{a}\left(\lambda^{w_{i}} x_{i}\right)
$$

where $x_{0}=t$ and $x_{i}$ are the coordinates in the Euclidean time and the spatial directions, respectively. If the action has non-trivial extrema, it should be stable under arbitrary variations of the fields. Thus, we can find a necessary condition for the existence of nontrivial extrema by looking at how the action behaves under the scaling eq. (2.2) with an arbitrary assignment of the scaling weights $w_{i}$.

Let $S_{\left(n_{0}, n_{1}, \cdots, n_{d}\right)}$ be the part of the action with \# $\#\left(\partial_{i}\right)=n_{i}$. For example, the standard kinetic term with two time derivatives (the first term in eq. (2.1)) is denoted by $S_{(2,0, \cdots, 0)}$. Under the transformation eq. $(2.2), S_{\left(n_{0}, n_{1}, \cdots, n_{d}\right)}$ scales as

$$
S_{\left(n_{0}, \cdots, n_{d}\right)} \rightarrow \lambda^{w_{\left(n_{0}, \cdots, n_{d}\right)}} S_{\left(n_{0}, \cdots, n_{d}\right)}, \quad w_{\left(n_{0}, \cdots, n_{d}\right)} \equiv \sum_{i=0}^{d}\left(n_{i}-1\right) w_{i}
$$

Here we assume that each term $S_{\left(n_{0}, \cdots, n_{d}\right)}$ is finite and positive definite. Then the stability of the action under this scaling requires that there should be at least one pair of terms 
whose scaling weights have opposite signs. This is the necessary condition for the existence of non-trivial solutions of the equations of motion.

Let us first recall the Lorentz symmetric case in two dimensions. For a generic assignment of the scaling weights, the standard kinetic terms $S_{(2,0)}$ and $S_{(0,2)}$ have the weights with opposite signs

$$
w_{(2,0)}=-w_{(0,2)}=w_{0}-w_{1} .
$$

Thus, the Lorentz symmetric scalar field theories in two dimensions can have non-trivial solutions ${ }^{1}$ which are stable under the transformations with $w_{0} \neq w_{1}$. On the other hand, for $w_{0}=w_{1}$, both $S_{(2,0)}$ and $S_{(0,2)}$ are scale invariant, so that the non-trivial configurations are marginally stable under the scaling with $w_{0}=w_{1}$. In other words, they have scale moduli which parameterize the set of marginally stable solutions. Such configurations with scale moduli are known as the sigma model instantons (a.k.a. the sigma model lumps). Their size is fixed when there are additional terms with opposite scaling weights in the action. The baby Skyrmions are such solitons with fixed sizes in the models with potential terms $S_{(0,0)}$ and higher derivative terms $S_{(m, n)}[40,41]$.

We can generalize the above discussion to arbitrary dimensions. In general, $S_{(2,0, \cdots, 0)}$ and $S_{(0,2, \cdots, 2)}$ have the scaling weights with opposite signs

$$
w_{(2,0, \cdots, 0)}=-w_{(0,2, \cdots, 2)}=w_{0}-\sum_{i=1}^{d} w_{i} .
$$

Thus, they are stable under the generic scaling transformations and invariant under any scaling transformaions with $w_{0}=\sum_{i=1}^{d} w_{i}$. Therefore, the action of the form

$$
S=S_{(2,0, \cdots, 0)}+S_{(0,2, \cdots, 2)},
$$

can have non-trivial configurations with multiple scale moduli. Such scale moduli are fixed if there are additional potential and higher derivative terms. In the following, we mainly consider instantons in scale invariant theories with the action of the form of eq. (2.6).

\section{2 "Supersymmetric" models and BPS equations}

In this section, we discuss the form of the Lifshitz-type action which admits instanton solutions. We focus on the BPS case in which the action is bounded below by the topological charge of instantons. Such an action, which satisfies the so-called "detailed balance condition", is characterized by a functional defined on each time slice

$$
W=\int d^{d} x \mathcal{W}\left(\phi, \partial_{i} \phi, \cdots\right),
$$

where $\mathcal{W}$ is a function of $\phi^{a}$ and their spatial derivatives. In terms of the functional $W$, the action of the non-linear sigma model which admits BPS instantons is given by

$$
S=\int d t d^{d} x\left[\frac{1}{2} g_{a b} \partial_{t} \phi^{a} \partial_{t} \phi^{b}+\frac{1}{2} g^{a b} \frac{\delta W}{\delta \phi^{a}} \frac{\delta W}{\delta \phi^{b}}\right],
$$

\footnotetext{
${ }^{1}$ Note that Derrick's theorem does not guarantee the existence of non-trivial solutions.
} 
where the variation of $W$ takes the form

$$
\frac{\delta W}{\delta \phi^{a}}=\frac{\partial \mathcal{W}}{\partial \phi^{a}}-\partial_{i} \frac{\partial \mathcal{W}}{\partial \partial_{i} \phi^{a}}+\{\text { higher derivative terms }\} .
$$

For simplicity, we assume that $\mathcal{W}$ does not contain the higher derivative of $\phi^{a}$ in the following. In $(d+1)$-dimensional spacetime with $d=0,1,2$, the action eq. (2.8) can be embeded into a supersymmetric model (see appendix A) which has a complex supercharge $Q$ satisfying the algebra

$$
\{Q, \bar{Q}\}=2 i \partial_{t}, \quad Q^{2}=\bar{Q}^{2}=0 .
$$

We call the functional $W$ a "superpotential" since it is a generalization of the superpotential in the standard supersymmetric theories. The supersymmetry in the Lifshitz-type field theory in higher dimensions will be discussed elsewhere.

The action eq. (2.8) can be rewritten into the Bogomol'nyi form

$$
S=T+\int d t d^{d} x \frac{1}{2} g_{a b}\left(\partial_{t} \phi^{a}+g^{a c} \frac{\delta W}{\delta \phi^{c}}\right)\left(\partial_{t} \phi^{b}+g^{b d} \frac{\delta W}{\delta \phi^{d}}\right) \geq T,
$$

where the topological charge $T$ takes the form

$$
T=\int d t d^{d} x\left[-\partial_{t} \mathcal{W}+\partial_{i}\left(\partial_{t} \phi^{a} \frac{\partial \mathcal{W}}{\partial \partial_{i} \phi^{a}}\right)\right] .
$$

Since the topological charge $T$ is a total derivative term, it has a fixed value for a given boundary condition. Therefore, the configurations with the least action in a fixed topological sector are the solutions of the following gradient flow equation

$$
\partial_{t} \phi^{a}=-g^{a b} \frac{\delta W}{\delta \phi^{b}} .
$$

This is the BPS equation which describes instantons in the Lifshitz-type sigma model.

Among various choices of the superpotential $W$, we focus on a specific class of $W$ which has several special symmetries. Let $\omega$ be a closed $(d+1)$-form on the target manifold $\mathcal{M}$

$$
\omega=\omega_{a_{1} \cdots a_{d+1}} d \phi^{a_{1}} \wedge \cdots \wedge d \phi^{a_{d+1}}, \quad d \omega=0 .
$$

Then, we can find a functional $W$ such that ${ }^{2}$

$$
\frac{\delta W}{\delta \phi^{a}}=\omega_{a b_{1} \cdots b_{d}} \partial_{x_{1}} \phi^{b_{1}} \cdots \partial_{x_{d}} \phi^{b_{d}} .
$$

${ }^{2}$ Since $\omega$ is a closed form, the superpotential

$$
W=\int d^{d} x \int_{-\infty}^{t} d t^{\prime} \omega_{a b_{1} \cdots b_{d}} \partial_{t^{\prime}} \phi^{a} \partial_{x_{1}} \phi^{b_{1}} \cdots \partial_{x_{d}} \phi^{b_{d}},
$$

depends only on the field on the boundary. If we assume that the fields $\phi^{a}$ approaches to a single point on the target space $\mathcal{M}$, the superpotential $W$ can be viewed as a functional defined on the time slice at $t$ whose variation gives eq. (2.15). 
The corresponding action takes the form

$$
S=S_{(2,0, \cdots, 0)}+S_{(0,2, \cdots, 2)}=\frac{1}{2} \int d t d^{d} x\left[\left|\partial_{t} \phi^{a}\right|^{2}+\left|g^{a b} \omega_{b c_{1} \cdots c_{d}} \partial_{1} \phi^{c_{1}} \cdots \partial_{d} \phi^{c_{d}}\right|^{2}\right],
$$

where $|\cdot|$ denotes the norm with respect to the metric $g_{a b}$. As we have discussed in the previous section, this type of action can have marginally stable instanton solutions. Their topological charge is given by

$$
T=\int d t d^{d} x \omega_{a b_{1} \cdots b_{d}} \partial_{t} \phi^{a} \partial_{x_{1}} \phi^{b_{1}} \cdots \partial_{x_{d}} \phi^{b_{d}}=\int_{\mathbb{R}^{d+1}} \phi^{*} \omega
$$

where $\phi^{*} \omega$ denotes the pullback of $\omega$ with respect to the map $\phi$ from the $(d+1)$-dimensional spacetime to the target space $\mathcal{M}$.

One of the most significant properties of the action eq. (2.16) is that it is invariant under the $z=d$ Lifshitz scaling transformation

$$
t \rightarrow \lambda^{d} t, \quad x_{i} \rightarrow \lambda x_{i}
$$

and the spatial volume-preserving diffeomorphism

$$
x_{i} \rightarrow x_{i}^{\prime}(x), \quad \operatorname{det}\left(\frac{\partial x_{i}^{\prime}}{\partial x_{j}}\right)=1 .
$$

As a consequence of these symmetry, instantons in this class of theories have continuous degeneracy associated with the symmetry broken by the configurations. The corresponding moduli parameters determines the shape of the instanton which can vary under the above transformations.

The action of the form eq. (2.16) has some exotic properties: for example, it vanishes for an arbitrary static configuration which does not depend on one of the spatial coordinates. To obtain a physically reasonable model, we should modify the action by adding terms which would break the stability of the instantons. In the presence of such a modification, instantons are no longer true minima of the action. Nevertheless, the path integral in the modified model is dominated by the so-called constrained instantons [42] whose leading order forms are given by the BPS configurations in the original model. Therefore, it is still important to know the properties of the instantons in the model eq. (2.16) and we will mainly focus on them in the following.

\subsection{Anisotropic BPS baby Skyrmions}

Let us first see the simplest example, the sigma model lump, in the Lorentz symmetric Kähler sigma model in two dimensions [43]. Then, we will see that it takes an anisotropic shape with a fixed size in the presence of additional potential and spatial higher derivative terms.

When the target space $\mathcal{M}$ is a Kähler manifold, the standard sigma model action

$$
S=\int d t d x g_{a \bar{b}}\left(\partial_{t} \phi^{a} \partial_{t} \bar{\phi}^{b}+\partial_{x} \phi^{a} \partial_{x} \bar{\phi}^{b}\right)
$$


can be obtained by setting

$$
W=\int d x \frac{i}{2}\left(\partial_{x} \phi^{a} \frac{\partial}{\partial \phi^{a}}-\partial_{x} \bar{\phi}^{a} \frac{\partial}{\partial \bar{\phi}^{a}}\right) K
$$

where $K$ is the Kähler potential which gives the target space metric $g_{a \bar{b}}=\frac{\partial^{2} K}{\partial \phi^{a} \partial \phi^{b}}$. For this superpotential, the BPS equation takes the form

$$
\partial_{t} \phi^{a}+g^{a \bar{b}} \frac{\delta W}{\delta \bar{\phi}^{b}}=\left(\partial_{t}+i \partial_{x}\right) \phi^{a}=0 .
$$

This is known as the BPS equation which describes the sigma model lumps. The corresponding topological charge is given by the pullback of the Kähler form [43]

$$
T=\int_{\mathbb{R}^{2}} \phi^{*}\left(i g_{a \bar{b}} d \phi^{a} \wedge d \bar{\phi}^{b}\right) .
$$

Since the solution to the BPS equations is an arbitrary holomorphic map $\phi^{a}=\phi^{a}(z)$, $(z=t+i x)$ we can freely change the scale $\phi^{a}(z) \rightarrow \phi^{a}(\lambda z)$ without changing the value of the action. This continuous parameter $\lambda$ is the size modulus associated with the scale invariance of the sigma model action.

As we have discussed in the previous section, the size of the BPS lump is fixed if we turn on a potential term and higher derivative terms. The baby Skyrmion is such a soliton with a fixed size in the Lorentz symmetric $\mathbb{C} P^{1}$ sigma model. In our setup, we can introduce spatial higher derivative terms $S_{(0, n)}(n>2)$ without breaking the BPS properties. An example of such model can be obtained by deforming $W$ as

$$
W=\int d x\left[\frac{i}{2}\left(\partial_{x} \phi^{a} \frac{\partial}{\partial \phi^{a}}-\partial_{x} \bar{\phi}^{a} \frac{\partial}{\partial \bar{\phi}^{a}}\right) K+\alpha g_{a \bar{b}} \partial_{x} \phi^{a} \partial_{x} \bar{\phi}^{b}+w(\phi, \bar{\phi})\right],
$$

where $\alpha$ is a constant parameter and $w(\phi, \bar{\phi})$ is a function on $\mathcal{M}$. Then, the BPS equation becomes

$$
\left(\partial_{t}+i \partial_{x}-\alpha \mathcal{D}_{x} \partial_{x}\right) \phi^{a}=-g^{a \bar{b}} \frac{\partial w}{\partial \bar{\phi}^{b}},
$$

where $\mathcal{D}_{x} \partial_{x} \phi^{a}=\partial_{x}^{2} \phi^{a}+\Gamma_{b c}^{a} \partial_{x} \phi^{b} \partial_{x} \phi^{c}$. Note that the scaling transformation $(t, x) \rightarrow(\lambda t, \lambda x)$ is no longer the symmetry of the BPS equation. Figure 1 shows a numerical solution of the anisotropic BPS configuration in the $\mathbb{C} P^{1}$ model with the Kähler potential $K=\log \left(1+|\phi|^{2}\right)$ and the superpotential

$$
W=\int d x\left[\frac{i}{2} \frac{\bar{\phi} \partial_{x} \phi-\phi \partial_{x} \bar{\phi}}{1+|\phi|^{2}}+\frac{\alpha\left|\partial_{x} \phi\right|^{2}+\beta}{\left(1+|\phi|^{2}\right)^{2}}\right]
$$

As shown in figure 1, the Lifshitz-type instanton has an anisotropic action density.

\subsection{Anisotropic BPS Skyrmions}

Next, we discuss the three dimensional Lifshitz-type non-linear sigma model whose target space is a Lie group $\mathcal{M}=G$. Since $\pi_{3}(G) \cong \mathbb{Z}$ for any compact simple Lie group, there 


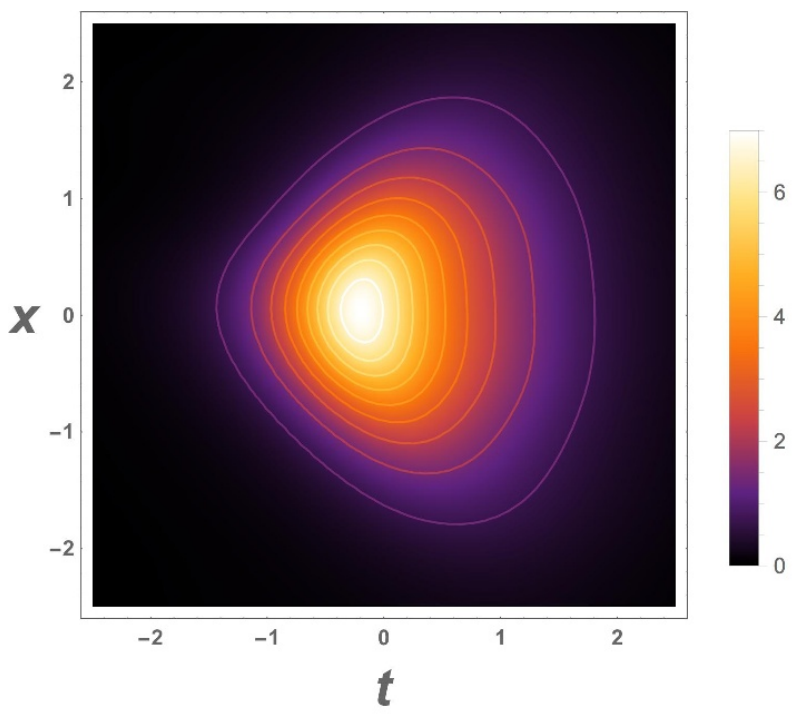

Figure 1. The anisotropic BPS baby Skyrmion (numerical solution): the action density for $\alpha=\frac{1}{4}$, $\beta=\frac{1}{2}$.

can be instantons characterized by the homotopy group $\pi_{3}(G)$. Such instantons are BPS configurations in the model with the superpotential which takes the form of the WessZumino-Witten term [38]

$$
W=\frac{1}{3} \int d^{2} x \int_{-\infty}^{t} d t i \epsilon_{I J K} \operatorname{Tr}\left[\left(i U^{\dagger} \partial_{I} U\right)\left(i U^{\dagger} \partial_{J} U\right)\left(i U^{\dagger} \partial_{K} U\right)\right], \quad(I, J, K=0,1,2) .
$$

This superpotential gives the following action with four spatial derivatives

$$
S=\frac{\xi}{2} \int d t d^{2} x \operatorname{Tr}\left\{\left(i U^{\dagger} \partial_{t} U\right)^{2}-\left[i U^{\dagger} \partial_{1} U, i U^{\dagger} \partial_{2} U\right]^{2}\right\}
$$

where $\xi$ is the inverse coupling constant. Since $\xi$ is dimensionless, this model is expected to be renormalizable according to the power-counting argument. The BPS equation takes the form

$$
i U^{\dagger} \partial_{t} U=i\left[i U^{\dagger} \partial_{1} U, i U^{\dagger} \partial_{2} U\right] .
$$

This system is invariant under the $z=2$ Lifshitz scaling and the spatial volume-preserving diffeomorphism. The topological charge is given by

$$
T=\frac{\xi}{3} \int d t d^{2} x i \epsilon^{I J K} \operatorname{Tr}\left[\left(i U^{\dagger} \partial_{I} U\right)\left(i U^{\dagger} \partial_{J} U\right)\left(i U^{\dagger} \partial_{K} U\right)\right] .
$$

We call the instanton in this model "anisotropic Skyrmion" since it is characterized by the same topological charge (baryon number) as the Skyrmion in the Lorentz symmetric theories.

\subsection{1 $G=\mathrm{SU}(2)$}

As an example, let us consider the case of $G=\mathrm{SU}(2)$. Let $(r, \theta)$ be polar coordinates of two-dimensional space. Combining the spatial rotation $\mathrm{SO}(2)$ with the $\mathrm{U}(1)_{V} \subset G_{L} \times G_{R}$ 
symmetry, we can construct the following $\mathrm{U}(1)$ symmetric ansatz

$$
U=V^{\dagger}\left(\begin{array}{cc}
\phi & -\sqrt{1-|\phi|^{2}} \\
\sqrt{1-|\phi|^{2}} & \bar{\phi}
\end{array}\right), \quad V=\exp \left(i k \theta \frac{\sigma_{3}}{2}\right),
$$

where $k$ is an integer and $\phi$ is a complex-valued function which is independent of the spatial angle coordinate $\theta .{ }^{3}$ Plugging this ansatz into the BPS equation eq. (2.29), we obtain the following equation for $\phi$ :

$$
\left[\partial_{t}+i \partial_{\rho}+\frac{i}{2}\left(\phi \partial_{\rho} \bar{\phi}-\bar{\phi} \partial_{\rho} \phi\right)\right] \phi=0
$$

where we have defined the spatial radial coordinate by $\rho=r^{2} / 4 k=\left(x_{1}^{2}+x_{2}^{2}\right) / 4 k$. Note that in this notation, the $z=2$ Lifshitz scaling transformation becomes

$$
t \rightarrow \lambda t, \quad \rho \rightarrow \lambda \rho .
$$

The scalar field $\phi$ can be viewed as a map from the $(t, \rho)$-plane (half plane $\rho \geq 0$ ) to the unit disk $|\phi| \leq 1$. If we assume that $\phi$ takes a fixed value at infinity, the $(t, \rho)$-plane can also be regarded as a disk whose boundary corresponds to the line $\rho=0$. Since the angle coordinate $\theta$ in the ansatz eq. (2.31) is ill-defined on the axis of the cylindrical coordinate system $(t, \rho, \theta)$, the scalar field $\phi$ should satisfy the boundary condition

$$
\lim _{\rho \rightarrow 0}|\phi|^{2}=1 .
$$

Then, the topological charge reduces to

$$
T=2 \pi k i \xi \int_{\rho \geq 0} d(\phi d \bar{\phi}-\bar{\phi} d \phi)=4 \pi k \xi \int_{\rho=0} d \arg \phi .
$$

This topological charge counts the winding number of the map $\phi$ from the line $\rho=0$ to the boundary $|\phi|=1$. Figure 2 shows a numerical solution of the BPS equation (2.32). There is a zero of $\phi$ on the $(t, \rho)$-plane around which $\arg \phi$ has a winding number. Note that the winding number and the integer $k$ are not individually identified with the topological invariant.

Once a BPS solution is obtained, we can find a continuous family of solutions by using the symmetries. The time translation and the Lifshitz scaling shift the zero of $\phi$, and hence its position on the $(t, \rho)$-plane can identified with a complex moduli parameter of the solution. We can also use the volume-preserving diffeomophism to generate a set of solutions. Let us focus on the $\mathrm{SL}(2, \mathbb{R})$ subgroup defined by

$$
\left(\begin{array}{l}
x_{1} \\
x_{2}
\end{array}\right) \rightarrow\left(\begin{array}{ll}
a & b \\
c & d
\end{array}\right)\left(\begin{array}{l}
x_{1} \\
x_{2}
\end{array}\right), \quad a d-b c=1 .
$$

Since the action of the spatial rotation $\mathrm{SO}(2) \subset \mathrm{SL}(2, \mathbb{R})$ is trivial for a rotationally symmetric solution, the $\mathrm{SL}(2, \mathbb{R})$ orbit of the solution is the hyperbolic plane $H^{2} \cong \mathrm{SL}(2, \mathbb{R}) / \mathrm{SO}(2)$. Therefore, the set of solutions has two real parameters corresponding to the coordinates of the hyperbolic plane.

\footnotetext{
${ }^{3}$ The same ansatz has been considered in Skyrmions trapped inside a vortex [44, 45] and fractional instantons on $\mathbb{R}^{2} \times S^{1}$ with a twisted boundary condition $[46,47]$.
} 


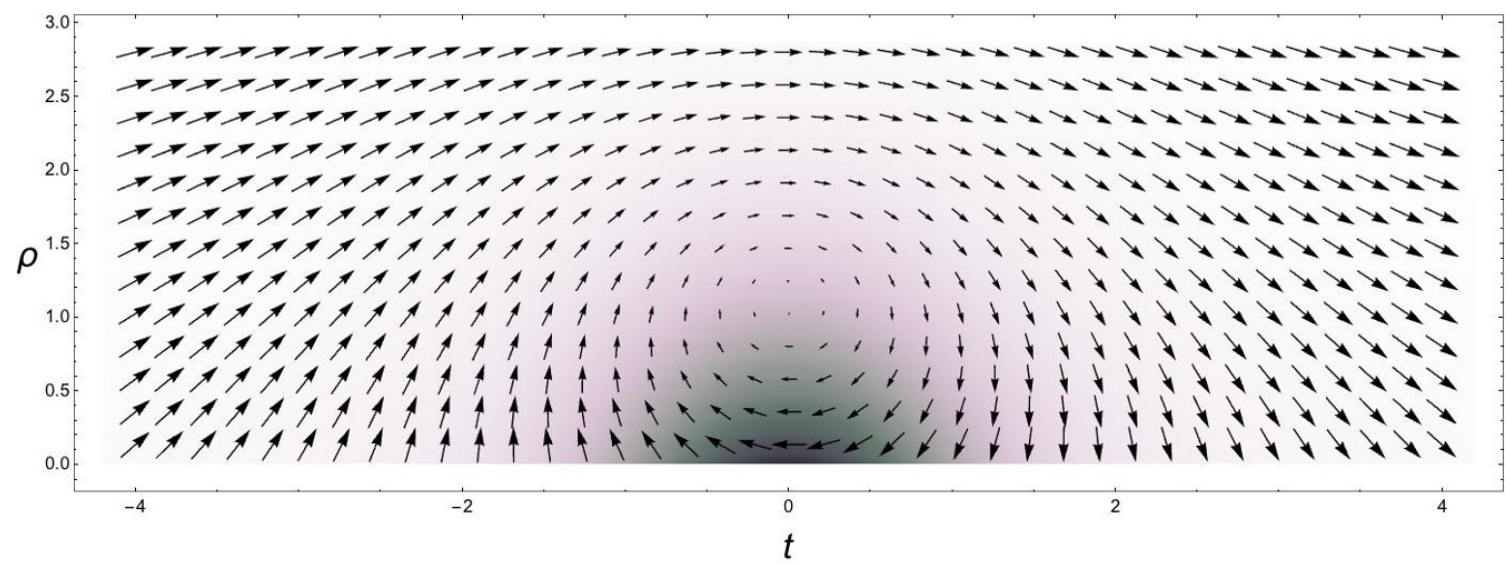

Figure 2. A numerical solution. The grayscale shows the action density and each vector indicates the scalar field $(\operatorname{Re} \phi, \operatorname{Im} \phi)$ at each point. The action density is localized at $t=0$ on the $t$-axis.

Deformation. We can also add the following term without breaking the $z=2$ Lifshitz scaling invariance

$$
\Delta W=\frac{\alpha}{2} \int d^{2} x \operatorname{Tr}\left(i U^{\dagger} \partial_{i} U\right)^{2} .
$$

The BPS equation becomes

$$
i U^{\dagger} \partial_{t} U=\left(\epsilon_{i j}+\alpha \delta_{i j}\right) \partial_{i}\left(i U^{\dagger} \partial_{j} U\right)
$$

For $\alpha \neq 0$, there is no longer the spatial volume-preserving diffeomorphism, and hence the $H^{2}$ moduli parameters are fixed. On the other hand, the zero of $\phi$ remains a complex moduli parameter since the time translation and the $z=2$ Lifshitz scaling symmetry are still unbroken.

We remark that the superpotential $W$ now takes the form of the Wess-Zumino-Witten action. As pointed out in [3], the ground state wavefuncitonal in the $(2+1)$ dimensional theory reproduces the partition function of the $2 \mathrm{~d}$ theory defined by the action $W$. A detailed study of this model would also be interesting.

Compactification. Let us next discuss instantons at finite temperature by compactifying the Euclidean time direction. In this case, we can consider an ansatz which is invariant under a combination of the time translation and the $\mathrm{U}(1)$ internal symmetry $\phi \rightarrow e^{i \alpha} \phi$. The exact BPS solution which has such a symmetry is given by

$$
\phi=\exp \left(2 \pi i n \frac{t+i \rho}{\beta}\right), \quad n \in \mathbb{Z} .
$$

The topological charge of this solution is independent of the inverse temperature $\beta$

$$
T=\frac{8 \pi^{2} n^{2} \xi}{\beta^{2}} \int_{0}^{\beta} d t \int d^{2} x \exp \left[-\frac{n \pi}{k \beta}\left(x_{1}^{2}+x_{2}^{2}\right)\right]=8 \pi^{2} n k \xi .
$$

Since we have compactified the time direction, the Lifshitz scaling $t \rightarrow \lambda^{2} t, x_{i} \rightarrow \lambda x_{i}$ is no longer a symmetry but a transformation which relates the solutions for different values 


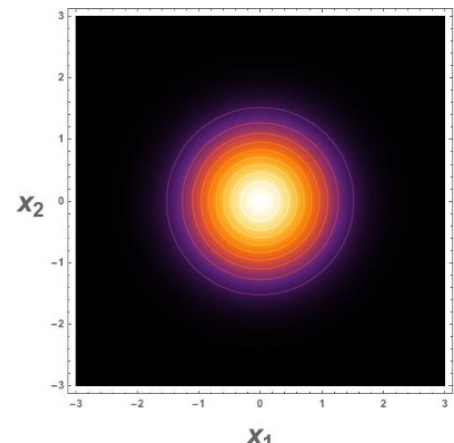

(a) $\vartheta=0$

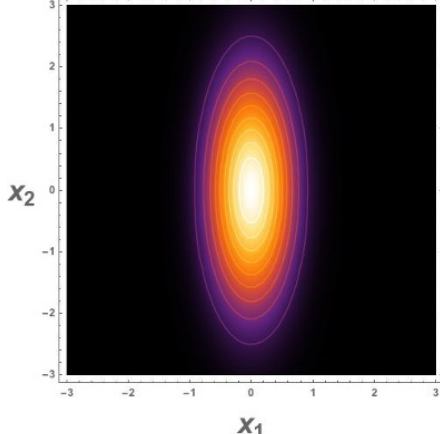

(b) $\vartheta=1, \varphi=0$

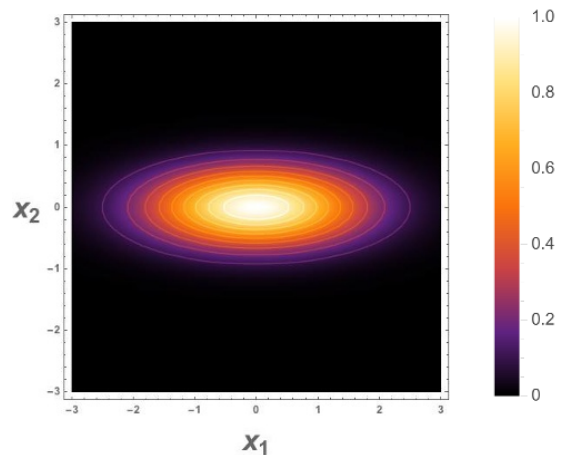

(c) $\vartheta=1, \varphi=\pi$

Figure 3. The action density on the $\left(x_{1}, x_{2}\right)$-plane.

of the inverse temperature $\beta$. On the other hand, there is still the the $\operatorname{SL}(2, \mathbb{R})$ symmetry eq. (2.36), under which the topological charge density in eq. (2.40) transforms in such a way that $x_{1}^{2}+x_{2}^{2}$ is replaced by

$$
x_{1}^{2}+x_{2}^{2} \rightarrow\left(x_{1}^{2}+x_{2}^{2}\right) \cosh \vartheta+\left(2 x_{1} x_{2}\right) \sinh \vartheta \sin \varphi+\left(x_{1}^{2}-x_{2}^{2}\right) \sinh \vartheta \cos \varphi,
$$

where $(\vartheta, \varphi)$ are moduli parameters corresponding to $\operatorname{SL}(2, \mathbb{R}) / \mathrm{SO}(2)$. Figure 3 shows the shape of the instanton for various values of the moduli parameters. The contour lines of the action density are ellipses and the parameters $(\vartheta, \varphi)$ are related to the eccentricity $e=\sqrt{1-e^{-2 \vartheta}}$ and the rotational angle, respectively.

The exact solution discussed above is symmetric under the time translation. In general, such a symmetric configuration takes the form

$$
\mathrm{U}(t, x)=e^{i v_{L} t} \mathrm{U}(x) e^{-i v_{R} t},
$$

where $v_{L}$ and $v_{R}$ are fixed elements of the Lie algebras $\mathfrak{g}_{L}$ and $\mathfrak{g}_{R}$, respectively. For this ansatz, eq. (2.29) becomes

$$
v_{R}-U^{\dagger} v_{L} U=i\left[i U^{\dagger} \partial_{1} U, i U^{\dagger} \partial_{2} U\right] .
$$

This is the BPS equation in the two dimensional system described by the action

$$
S=\frac{\xi}{2} \int d^{2} x \operatorname{Tr}\left\{\left(v_{R}-U^{\dagger} v_{L} U\right)^{2}-\left[i U^{\dagger} \partial_{1} U, i U^{\dagger} \partial_{2} U\right]^{2}\right\} .
$$

This action is bounded below by the topological charge

$$
T=\xi \int_{\mathbb{R}^{2}} d\left\{\operatorname{Tr}\left(i v_{L} U d U^{\dagger}\right)-\operatorname{Tr}\left(i v_{R} U^{\dagger} d U\right)\right\} .
$$

If the vacua $\mathcal{M}_{\text {vac }}=\left\{U \in G \mid v_{R}=U^{\dagger} v_{L} U\right\}$ has a non-trivial first homotopy group $\pi_{1}\left(\mathcal{M}_{\text {vac }}\right) \neq 1$, there can be global vortex configurations characterized by the boundary condition [47]

$$
\lim _{r \rightarrow \infty} \mathrm{U}(x)=U_{0} e^{i v \theta}
$$


where $U_{0} \in G$ is a solution of the vacuum condition $v_{R}=U^{\dagger} v_{L} U$ and $v$ is an element of $\mathfrak{g}_{R}$ such that $\left[v, v_{R}\right]=0$ and $e^{2 \pi v i}=\mathbf{1}$. For a BPS configuratioin satisfying the above boundary condition, the topological charge eq. (2.45) is given by

$$
T=4 \pi \xi \operatorname{Tr}\left(v_{R} v\right) .
$$

Note that although the action (energy) for a global vortex configuration is logarithmically divergent in the presence of the standard kinetic terms [47], it is finite in this type of model which has only potential and higher derivative terms.

\subsection{Instantons in higher dimensional sigma models}

\subsection{1 $O(d+1)$ sigma model}

In the previous section, we have considered the non-linear sigma model with $\mathcal{M}=\mathrm{SU}(2) \cong$ $S^{3}$ in three dimensions. As a generalization to higher dimensions, we can consider the $(d+1)$-dimensional models with $\mathcal{M}=S^{d+1}$, i.e. the $O(d+1)$ sigma model. The action which admits BPS instantons takes the form

$$
S=\frac{\xi}{2} \int d t d^{d} x\left[\partial_{t} \vec{n} \cdot \partial_{t} \vec{n}+\vec{\Omega} \cdot \vec{\Omega}\right], \quad \Omega^{a}=\epsilon^{a b c_{1} \cdots c_{d}} n^{b} \partial_{1} n^{c_{1}} \cdots \partial_{d} n^{c_{d}},
$$

where $\vec{n}$ is a unit vector $\vec{n} \cdot \vec{n}=1$. The Bogomol'nyi bound for this action is given by the following topological charge corresponding to $\pi_{d+1}\left(S^{d+1}\right)=\mathbb{Z}$

$$
S \geq T=\xi \int d t d^{d} x \partial_{t} \vec{n} \cdot \vec{\Omega}=\xi \int_{\mathbb{R}^{d+1}} \phi^{*} \operatorname{Vol}\left(S^{d+1}\right),
$$

where $\phi^{*} \operatorname{Vol}\left(S^{d+1}\right)$ is the pullback of the volume form on $S^{d+1}$. The BPS equation is

$$
\partial_{t} \vec{n}=\vec{\Omega} .
$$

Let us assume that the single instanton solution is symmetric under the spatial rotation $\mathrm{SO}(d)$. Combining with the $\mathrm{SO}(d)$ symmetry of the target space, we can find the following hedgehog ansatz

$$
n^{a}=\sqrt{1-|\phi|^{2}} \frac{x^{a}}{r} \quad(a=1, \cdots, d), \quad n^{d+1}+i n^{d+2}=\phi,
$$

where $r$ is the spatial radial coordinate. The condition for the smoothness of the configuration at $r=0$ implies that the complex-valued function $\phi$ should satisfy the boundary condition

$$
\lim _{r \rightarrow 0}|\phi|=1
$$

Substituting the ansatz into eq. (2.50), we obtain the BPS equation for $\phi$

$$
\partial_{t} \phi=-\left(1-|\phi|^{2}\right)^{\frac{d-2}{2}}\left[i \partial_{\rho}+\frac{i}{2}\left(\phi \partial_{\rho} \bar{\phi}-\bar{\phi} \partial_{\rho} \phi\right)\right] \phi,
$$

where we have redefined the spatial radial coordinate by $\rho=r^{d} / d$. Note that this BPS equation reduces to eq. (2.32) for $d=2$. The topological charge becomes

$$
T=\frac{V_{d-1}}{d} \xi \int_{\rho=0} d \arg \phi .
$$

As in the case of $\mathcal{M}=\mathrm{SU}(2)$, this topological charge counts the winding number of the map from the line $\rho=0$ to the boundary of the disk $|\phi|=1$. 
Compactification. Assuming that the Euclidean time direction is compact, we can obtain the following implicit form of an exact solution which is symmetric under the time translation

$$
\phi=\sqrt{1-f(\rho)^{2}} e^{\frac{2 \pi i t}{\beta}}, \quad \sqrt{(1-f)(1+f)^{(-1)^{d}}}=\exp \left(-\frac{2 \pi}{\beta} \rho-\sum_{l=1}^{\left\lfloor\frac{d-1}{2}\right\rfloor} \frac{f^{d-2 l}}{d-2 l}\right),
$$

where $\lfloor x\rfloor$ denotes the largest integer not greater than $x$. For any $d$, the scalar field $\phi$ has the asymptotic form

$$
\phi \simeq \exp \left[\frac{2 \pi i}{\beta}(t+i \rho)\right], \quad(\rho \rightarrow \infty) .
$$

The scalar field $\phi$ vanishes at the spatial infinity, so that $\left.\vec{n}\right|_{\rho \rightarrow \infty}$ defines a map from $S_{\infty}^{d-1}$ to $S^{d-1}=\left\{\vec{n} \in S^{d+1} \mid \phi=0\right\}$. Therefore, this configuration is a global type soliton characterized by $\pi_{d-1}\left(S^{d-1}\right)$.

\subsubsection{Other examples}

Another class of higher dimensional models which admits BPS instantons is the Kähler sigma models. Since any Kähler manifold has the closed forms

$$
\omega^{l}=\left(i g_{a \bar{b}} d \phi^{a} \wedge d \bar{\phi}^{b}\right)^{l}, \quad\left(l=1, \cdots, \operatorname{dim}_{\mathbb{C}} \mathcal{M}\right)
$$

there can be instantons characterized by the topological charge of the form

$$
T=\xi \int_{\mathbb{R}^{d+1}} \phi^{*} \omega^{n+1}, \quad(d=2 n+1) .
$$

This topological charge gives the BPS bound for the action

$$
S=\frac{\xi}{2} \int d t d^{d} x g_{a \bar{b}}\left[\partial_{t} \phi^{a} \partial_{t} \bar{\phi}^{b}+\left(V^{i} \partial_{i} \phi^{a}\right) \overline{\left(V^{i} \partial_{i} \phi^{b}\right)}\right]
$$

where $V^{i}$ is defined by

$$
V^{i}=\epsilon_{i j_{1} \cdots j_{2 n}}\left(\phi^{*} \omega\right)_{j_{1} j_{2}} \cdots\left(\phi^{*} \omega\right)_{j_{2 n-1} j_{2 n}}, \quad\left(\phi^{*} \omega\right)_{i j}=i g_{b \bar{c}} \partial_{i} \phi^{a} \partial_{j} \bar{\phi}^{b} .
$$

The BPS equation

$$
\left(\partial_{t}+i V^{i} \partial_{i}\right) \phi^{a}=0
$$

can be regarded as a generalization of that in $(1+1)$-dimensional Kähler sigma models in eq. (2.22).

When the target space $\mathcal{M}$ is a Calabi-Yau manifold of complex dimension $d+1$, (the real part of) the holomorphic $(d+1)$-form can also be used as the superpotential. In general, any calibration form on a calibrated manifold can be used to construct a nonlinear sigma model with BPS instantons. It would also be interesting to consider instantons corresponding to closed forms generated by the triplet of Kähler forms on hyper-Kähler manifolds. 


\section{Instantons in Lifshitz-type gauge theories}

In this section, we discuss instantons in the Lifshitz-type gauge theories. As is well-known, there exist instanton solutions in the pure Yang-Mills theory in the $(3+1)$-dimensional spacetime. In the following, we generalize the Yang-Mills instantons to the $(d+1)$ dimensional gauge theories with the classical $z=d-2$ Lifshitz scaling symmetry.

\subsection{Derrick's scaling argument in Lifshitz-type gauge theories}

First, we discuss the stability of instantons by generalizing the scaling argument in section 2.1 to the case of gauge fields. Since gauge fields are associated with covariant derivatives, we assign the scaling weights so that each component $A_{i}$ has the same scaling properties as the derivative $\partial_{i}$. Then, the covariant derivatives transform under the scaling eq. (2.2) as

$$
\partial_{i}+i A_{i} \rightarrow \lambda^{-w_{i}}\left(\partial_{i}+i A_{i}\right)
$$

If an action for the gauge field have non-trivial extrema, it should be stable under the scaling transformation with arbitrary weights. In four dimensions, the electric and the magnetic parts of the Yang-Mills action $S_{\mathrm{YM}}$

$$
S_{E_{i}}=\frac{1}{2 g^{2}} \int d t d^{3} x E_{i}^{2}, \quad S_{B_{i}}=\frac{1}{2 g^{2}} \int d t d^{3} x B_{i}^{2},
$$

have the scaling weights with the opposite signs $w\left(S_{E_{i}}\right)=-w\left(S_{B_{i}}\right)=w_{0}+w_{i}-\sum_{j=1, j \neq i}^{3} w_{j}$ for generic $w_{i}(i=0,1,2,3)$, whereas both terms are scale invariant for $w_{0}=w_{i}(i=1,2,3)$. Therefore, the Yang-Mills instanton is stable under the generic scaling and marginally stable for the scaling with $w_{0}=w_{i}$.

As a generalization to higher dimensions, we can consider an analogue of eq. (3.2). For $d=2 n+1$, define $E_{i}$ and $B_{i}$ by

$$
E_{i}=F_{t i}, \quad B_{i}=\frac{1}{2^{n}} \epsilon_{i j_{1} \cdots j_{2 n}} F_{j_{1} j_{2}} \cdots F_{j_{2 n-1} j_{2 n}} .
$$

Since $S_{E_{i}}$ and $S_{B_{i}}$ have the opposite weights

$$
w\left(S_{E_{i}}\right)=-w\left(S_{B_{i}}\right)=w_{0}+w_{i}-\sum_{j=1, j \neq i}^{d} w_{j},
$$

there can be instanton solutions in the higher dimensional gauge theory described by

$$
S=\frac{1}{2 g^{2}} \int d t d^{d} x \operatorname{Tr}\left(E_{i}^{2}+B_{i}^{2}\right)
$$

Since this action is invariant under the $z=d-2$ Lifshitz scaling $\left(w_{0}=d-2, w_{i}=1\right.$ $(i=1, \cdots, d))$, the instantons are marginally stable and hence they have associated size moduli parameters. Note that the gauge coupling constant $g$ is dimensionless and hence this model is expected to be renormalizable according to the power-counting argument. 


\subsection{Weyl rescaling and coset space dimensional reduction}

An important property of the action eq. (3.5) is that it is invariant under a generalized version of the Weyl transformation. Let us put the model on a curved space with a metric of the form

$$
d s^{2}=N^{2} d t^{2}+g_{i j}\left(d x^{i}+N^{i} d t\right)\left(d x^{j}+N^{j} d t\right) .
$$

The covariantized action ${ }^{4}$ is invariant under the following change of the metric

$$
N \rightarrow \lambda\left(t, x_{i}\right)^{d-2} N, \quad g_{i j} \rightarrow \lambda\left(t, x_{i}\right)^{2} g_{i j} .
$$

Using this anisotropic Weyl transformation with $\lambda=r^{-1}$, we can map the flat spacetime to the direct product of the hyperbolic plane and the $(d-1)$-dimensional sphere $H^{2} \times S^{d-1}$

$$
d s^{2}=d t^{2}+d r^{2}+r^{2} d \Omega_{d-1}^{2} \sim \frac{1}{(d-2)^{2}} \frac{d t^{2}+d \rho^{2}}{\rho^{2}}+d \Omega_{d-1}^{2},
$$

where $\rho=r^{d-2} /(d-2)$. Therefore, the theory can be reduced to a two-dimensional system defined on the hyperbolic plane $H^{2}$ by taking an ansatz which is symmetric under a combination of the $\mathrm{SO}(d)$ rotation and the gauge transformation. It is known that the self-dual equation in the four dimensional pure Yang-Mills theory $(d=3)$ reduces to the vortex equation on the hyperbolic plane [48]. This type of the dimensional reduction is called the coset space dimensional reduction [49].

We have already seen the examples of the coset space dimensional reduction for scalar fields (zero forms) in the previous section. In the following, we will see that the $\mathrm{SO}(d)$ symmetric instantons in the Lifshitz-type gauge theories can also be mapped to vortices on the hyperbolic plane by the dimensional reduction.

\subsection{Generalized Yang-Mills instantons}

As in the case of scalar field theories, the superpotential formalism (detailed balance condition) can be used to construct actions which admit BPS configurations. The scale invariant action eq. (3.5) for the $\mathrm{SU}(N)$ gauge field $A$ can be obtained by using the Chern-Simons form as the superpotential

$$
W=\frac{1}{n+1} \int_{\mathbb{R}^{d}} C S_{d}(A), \quad d C S_{d}=\operatorname{Tr} \underbrace{(F \wedge \cdots \wedge F)}_{n+1} .
$$

The corresponding topological charge is given by

$$
T=\frac{1}{g^{2}} \int d t d^{d} x \operatorname{Tr}\left(E_{i} B_{i}\right)=\frac{1}{(n+1) g^{2}} \int_{\mathbb{R}^{d+1}} \operatorname{Tr}(F \wedge \cdots \wedge F) .
$$

\footnotetext{
${ }^{4}$ The action which has covariance under the foliation-preserving diffeomorphism $t \rightarrow t^{\prime}(t)$ and $x_{i} \rightarrow$ $x_{i}^{\prime}\left(t, x_{j}\right)$ takes the form

$$
S=\int d t d^{d} x N \sqrt{\operatorname{det} g_{i j}} g^{i j}\left(E_{i} E_{j}+B_{i} B_{j}\right),
$$
}

where $E_{i}$ and $B_{i}$ are given by

$$
E_{i}=\frac{1}{N}\left(F_{t i}-N^{j} F_{j i}\right), \quad B_{i}=\frac{1}{2^{n}} \frac{1}{\sqrt{\operatorname{det} g_{i j}}} g_{i k} \epsilon^{k j_{1} \cdots j_{2 n}} F_{j_{1} j_{2}} \cdots F_{j_{2 n-1} j_{2 n}} .
$$


This gives the BPS bound for the action

$$
S \geq T+\frac{1}{2 g^{2}} \int d t d^{d} x \operatorname{Tr}\left(E_{i}-B_{i}\right)^{2} .
$$

The BPS equation is $E_{i}=B_{i}$, that is,

$$
F_{t i}=\frac{1}{2^{n}} \epsilon_{i j_{1} \cdots j_{2 n}} F_{j_{1} j_{2}} \cdots F_{j_{2 n-1} j_{2 n}} .
$$

This is a generalization of the self-dual equation in the four dimensional Yang-Mills theory.

\subsection{1 (5+1)-dimensional SU(4) gauge theory}

Let us consider the simplest example in the $(5+1)$-dimensional $\mathrm{SU}(4)$ gauge theory, in which we can consider the $\mathrm{SO}(5) \subset \mathrm{SU}(4)$ invariant ansatz. The action takes the form

$$
S=\frac{1}{2 g^{2}} \int d t d^{5} x \operatorname{Tr}\left[\left(F_{t i}\right)^{2}+\left(\frac{1}{4} \epsilon^{i j k l m} F_{j k} F_{l m}\right)^{2}\right] .
$$

This model has the classical $z=3$ Lifshitz scale invariance

$$
\left(\partial_{t}+i A_{t}\right) \rightarrow \lambda^{-3}\left(\partial_{t}+i A_{t}\right), \quad\left(\partial_{i}+i A_{i}\right) \rightarrow \lambda^{-1}\left(\partial_{i}+i A_{i}\right) .
$$

The BPS equation and the topological charge are given by

$$
F_{t i}=\frac{1}{4} \epsilon_{i j k l m} F_{j k} F_{l m}, \quad T=\frac{1}{3 g^{2}} \int_{\mathbb{R}^{6}} \operatorname{Tr}[F \wedge F \wedge F] .
$$

Let us consider configurations which are invariant under a combination of the spatial $\mathrm{SO}(5)$ rotation and an $\mathrm{SO}(5)$ subgroup in the $\mathrm{SU}(4)$ gauge group. The adjoint representation $\mathbf{1 5}$ of SU(4) can be decomposed into the vector representation $\mathbf{5}$ and the antisymmetric representation $\mathbf{1 0}$ of the $\mathrm{SO}(5)$ subgroup. Correspondingly, the $\mathfrak{s u}(4)$ generators $t^{a}(a=1, \cdots, 15)$ are decomposed into the 5 d gamma matrices and the $\mathrm{SO}(5)$ generators

$$
t^{a} \rightarrow \Gamma_{i}, \quad \Gamma_{i j}=\frac{i}{2}\left[\Gamma_{i}, \Gamma_{j}\right],
$$

where our convention for the gamma matrices is $\left\{\Gamma_{i}, \Gamma_{j}\right\}=2 \delta_{i j}$ and $\Gamma_{i}^{\dagger}=\Gamma_{i}$. Then, we can find the following basis of $\mathrm{SO}(5)$ invariant one-forms

$$
\omega_{t}=\Gamma_{i} \hat{x}^{i} d t, \quad \omega_{r}=\Gamma_{i} \hat{x}^{i} d r, \quad \omega_{ \pm}=\left[\Gamma_{i j} \hat{x}^{i} \pm i\left(\delta_{i j}-\hat{x}_{i} \hat{x}_{j}\right) \Gamma_{i}\right] d x^{j},
$$

where $r$ is the spatial radial coordinate and $\hat{x}_{i}=x_{i} / r$. In terms of the $\mathrm{SO}(5)$ invariant one-forms, the most general $\mathrm{SO}(5)$ symmetric ansatz for the gauge field is given by

$$
A=\frac{1}{2}\left(a_{t} \omega_{t}+a_{r} \omega_{r}+\frac{\phi-1}{2 r} \omega_{+}+\frac{\bar{\phi}-1}{2 r} \omega_{-}\right)
$$

where $a_{t}, a_{r}$ and $\phi$ are functions of $t$ and $r$. For this gauge field, the field strength takes the form

$$
F=\frac{1}{2}\left[\Gamma_{i} \hat{x}^{i} f+\frac{1}{2 r}\left(\mathcal{D} \phi \wedge \omega_{+}+\overline{\mathcal{D} \phi} \wedge \omega_{-}\right)+\frac{i}{8 r^{2}}\left(|\phi|^{2}-1\right)\left(\omega_{+} \wedge \omega_{-}+\omega_{-} \wedge \omega_{+}\right)\right],
$$


where $f$ is the two-dimensional field strength and $\mathcal{D} \phi$ is the covariant derivative defined by

$$
f=\left(\partial_{t} a_{r}-\partial_{r} a_{t}\right) d t \wedge d r, \quad \mathcal{D} \phi=\left(\partial_{t}+i a_{t}\right) \phi d t+\left(\partial_{r}+i a_{r}\right) \phi d r .
$$

Plugging into the BPS equation, we obtain the follwoing reduced equations for the two dimensional fields $a$ and $\phi$ :

$$
9 \rho^{2} f_{t \rho}+\left(|\phi|^{2}-1\right)^{2}=0, \quad \mathcal{D}_{t} \phi-i\left(|\phi|^{2}-1\right) \mathcal{D}_{\rho} \phi=0
$$

where we have redefined the spatial radial coordinate by $\rho=r^{3} / 9$. These equations can be regarded as the vortex equations in the following system defined on the hyperbolic plane

$$
S=\frac{3 V_{4}}{2 g^{2}} \int d t d \rho G\left[f_{t \rho} f^{t \rho}+4\left(\mathcal{D}_{t} \phi\right) \overline{\left(\mathcal{D}^{t} \phi\right)}+4\left(|\phi|^{2}-1\right)^{2}\left(\mathcal{D}_{\rho} \phi\right) \overline{\left(\mathcal{D}^{\rho} \phi\right)}+\left(|\phi|^{2}-1\right)^{4}\right],
$$

where $V_{4}$ is the volume of the unit 4-sphere and the spacetime indices are raised with the hyperbolic metric $G$

$$
d s^{2}=G\left(d t^{2}+d \rho^{2}\right)=\frac{d t^{2}+d \rho^{2}}{9 \rho^{2}} .
$$

This action can be obtained by substituting the rotationally symmetric ansatz eq. (3.19) into the original action eq. (3.14). Similarly, the 5d Chern-Simons term reduces to the superpotential for the two-dimensional system

$$
W=-\int d \rho\left[a_{\rho}+\frac{i}{2}\left(|\phi|^{2}-2\right)\left(\phi \mathcal{D}_{\rho} \bar{\phi}-\bar{\phi} \mathcal{D}_{\rho} \phi\right)\right] .
$$

In terms of this superpotential, the two-dimensional action can be rewritten as

$$
S=T+\frac{3 V_{4}}{2 g^{2}} \int d t d \rho\left[G\left(G^{-1} f_{t \rho}-\frac{\delta W}{\delta a_{\rho}}\right)^{2}+\left|2 \mathcal{D}_{t} \phi-\frac{\delta W}{\delta \bar{\phi}}\right|^{2}\right],
$$

where the topological charge is given by the first Chern number (up to trivial boundary terms)

$$
T=-\frac{3 V_{4}}{g^{2}} \int_{H^{2}} f
$$

The two-dimensional system is anisotropic in the sense that the action eq. (3.23) is not invariant under the whole $\mathrm{SL}(2, \mathbb{R})$ isometry of the hyperbolic plane. It is invariant under the subgroup of the isometry corresponding to the time translation and the Lifshitz scaling. These two symmetries shift the zeros of $\phi$ on the $(t, \rho)$-plane, which correspond to the positions and the size moduli of the $6 \mathrm{~d}$ instantons.

It has been shown in [48] that the $\mathrm{SO}(3)$ invariant instantons ${ }^{5}$ in the $4 \mathrm{~d} \mathrm{SU}(2)$ YangMills theory are described by the hyperbolic vortex equations in the two-dimensional system characterized by

$$
G=\frac{1}{\rho^{2}}, \quad W=-2 \int d \rho\left[a_{\rho}-\frac{i}{2}\left(\phi \mathcal{D}_{\rho} \bar{\phi}-\bar{\phi} \mathcal{D}_{\rho} \phi\right)\right] .
$$

Figure 4 shows the action densities of the hyperbolic vortices corresponding to $4 \mathrm{~d}$ and $6 \mathrm{~d}$ instantons. The profile of the $4 \mathrm{~d}$ instanton is invariant under the $\mathrm{SO}(2)$ subgroup of 


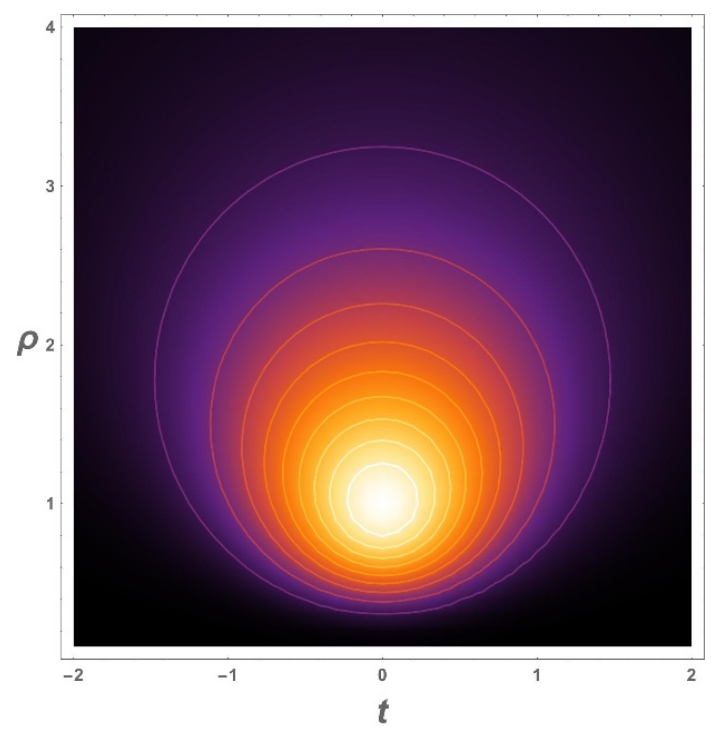

(a) 4 d instanton

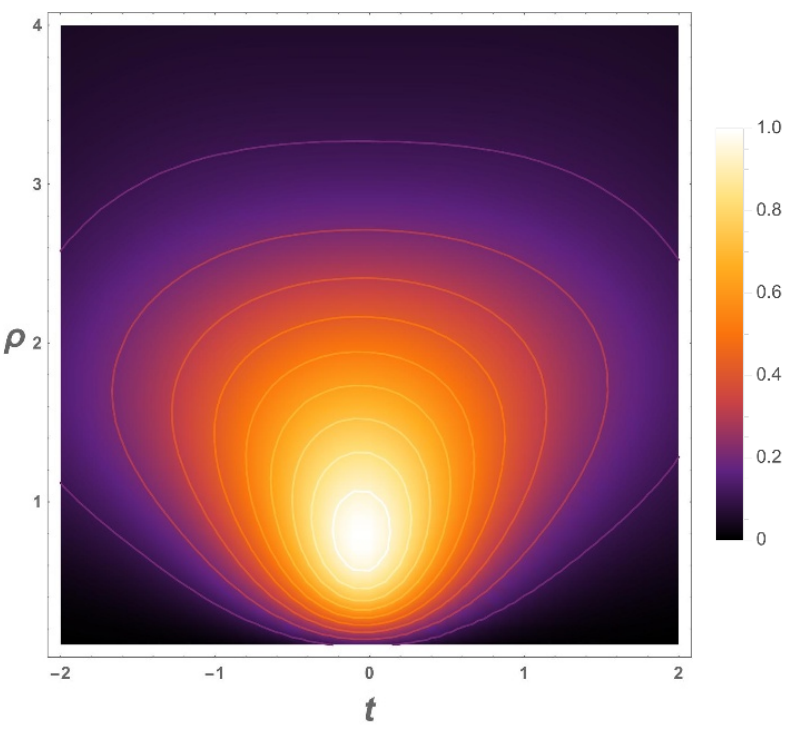

(b) $6 \mathrm{~d}$ instanton

Figure 4. The action densities of the hyperbolic vortices (excluding the volume element of the hyperbolic plane). Note that the action densities for the instantons, which can be obtained by multiplying the volume element, are localized around $t=0$ on the $t$-axis.

the $\mathrm{SL}(2, \mathbb{R})$ isometry whereas that of the $6 \mathrm{~d}$ instanton is not symmetric, reflecting the anisotropy of the original system.

Compactification. Next, let us consider the finite-temperature case. In the standard four dimensional $\mathrm{SU}(N)$ Yang-Mills theory, instantons at finite temperature are called calorons [50-52]. It is known that in the presence of a non-trivial holonomy around the Euclidean time circle, each caloron has $N$ constituents which can be identified with magnetic monopoles [53-57]. Here, we look for an analogous object which constitutes the instanton in our setup.

Since the finite-temperature system has the additional U(1) symmetry corresponding to the time translation, we can consider an ansatz which is invariant under the $\mathrm{U}(1) \times \mathrm{SO}(5)$ transformations. By appropriately choosing the gauge, we can find the following ansatz

$$
\phi=\bar{\phi}=\varphi(\rho), \quad a_{t}=a(\rho), \quad a_{\rho}=0 .
$$

Then, the BPS equations eq. (3.22) reduces to

$$
9 \rho^{2} a^{\prime}(\rho)=\left(\varphi^{2}-1\right)^{2}, \quad \varphi^{\prime}(\rho)=\frac{a \varphi}{\varphi^{2}-1} .
$$

The smoothness of the ansatz eq. (3.19) at $\rho=0$ requires that the profile functions $\varphi(\rho)$ and $a(\rho)$ should satisfy the boundary conditions

$$
\varphi(\rho=0)=1, \quad a(\rho=0)=0 .
$$

\footnotetext{
${ }^{5}$ The ansatz for the $\mathrm{SO}(3)$ invariant instantons in the $4 \mathrm{~d} \mathrm{SU}(2)$ Yang-Mills theory is given by eq. (3.19) with $\Gamma_{i}$ replaced by the Pauli matrices.
} 


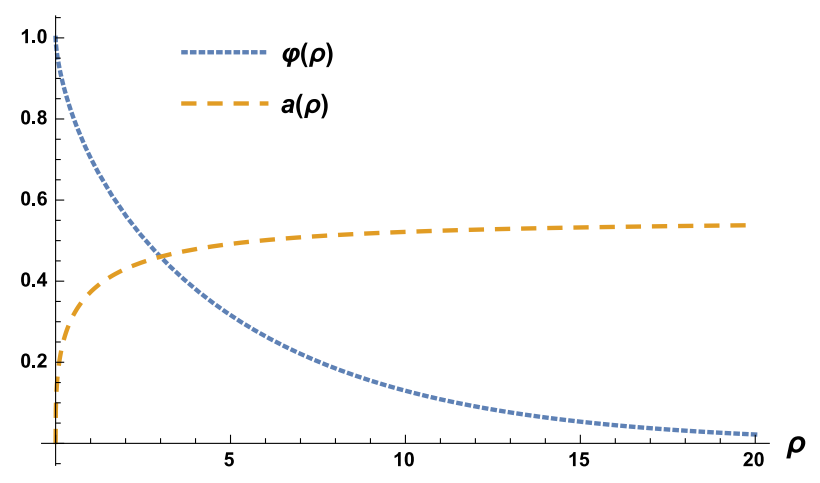

Figure 5. A numerical solution.

On the other hand, $a(\infty)$ is a free parameter which determines the value of the Polyakov loop at the spatial infinity. For $a(\infty) \neq 0$, the $\mathrm{SU}(4)$ gauge symmetry is broken to $\mathrm{SU}(2) \times$ $\mathrm{SU}(2) \times \mathrm{U}(1)$. A numerical solution is shown in figure 5 .

The time independent solution discussed above can be identified with the BPS soliton in the following five dimensional system which can be obtained by the dimensional reduction along the periodic time direction:

$$
S=\frac{1}{2 g^{2}} \int d^{5} x \operatorname{Tr}\left[\left(\mathcal{D}_{i} \Phi\right)^{2}+B_{i}^{2}\right], \quad B_{i}=\frac{1}{4} \epsilon_{i j k l m} F_{j k} F_{l m},
$$

where $\Phi$ is the adjoint scalar field corresponding to $A_{t}$. The topological charge of the BPS soliton in this system is given by

$$
T=\frac{1}{2 g^{2}} \int_{\mathbb{R}^{5}} \operatorname{Tr}\left(d_{A} \Phi \wedge F \wedge F\right) .
$$

The generalized monopole configurations characterized by this topological charge has been discussed in [58].

We can also perform the dimensional reduction along one of the spatial directions instead of the time direction. The resulting model is a five dimensional $z=3$ Lifshitz-type gauge theory with an adjoint scalar. It is characterized by the superpotential

$$
W=\int_{\mathbb{R}^{4}} \operatorname{Tr}(\Phi F \wedge F) .
$$

The corresponding action takes the form

$$
S=\frac{1}{2 g^{2}} \int d t d^{4} x \operatorname{Tr}\left[\left(F_{t i}\right)^{2}+\left(\mathcal{D}_{t} \Phi\right)^{2}+\left(\frac{1}{4} \epsilon_{i j k l} F_{i j} F_{k l}\right)^{2}+\left(\frac{1}{2} \epsilon_{i j k l}\left\{\mathcal{D}_{j} \Phi, F_{k l}\right\}\right)^{2}\right] .
$$

This gauge theory is super-renormalizable since the coupling constant has the positive dimension $\left[g^{2}\right]=\left[\partial_{x}\right]$. The BPS soliton in this system has the same topological charge as eq. (3.33) and obeys the BPS equations

$$
\mathcal{D}_{t} \Phi=\frac{1}{4} \epsilon_{i j k l} F_{i j} F_{k l}, \quad F_{t i}=\frac{1}{2} \epsilon_{i j k l}\left\{\mathcal{D}_{j} \Phi, F_{k l}\right\} .
$$

This equation can also be viewed as a generalization of the BPS monopole equation. 


\section{Summary and discussion}

In this paper, we have discussed BPS instantons in the Lifshitz-type non-linear sigma models and gauge theories. In the "supersymmetric models" in which the detailed balance condition is satisfied, the BPS equations for the instantons are described by the gradient flow equations for the "superpotential" $W$. For the Lifshitz-type non-linear sigma models, we have focused on BPS instantons in the $(d+1)$-dimensional settings where the action is invariant under the $z=d$ Lifshitz scaling and the spatial volume-preserving diffeomorphism. As examples, the anisotropic BPS (baby) Skyrmions and their higher dimensional generalizations have been discussed in the Lifshitz-type $O(d+1)$ sigma models. For the Lifshitz-type gauge theories, we have discussed a generalization of the Yang-Mills instanton to the higher dimensional theories. The anisotropic Weyl rescaling and the coset space dimensional reduction have been used to map the instantons to Abelian vortices in the anisotropic system on the hyperbolic plane. As in the case of the instantons in four dimensions $[59,60]$, it is also interesting to consider more general dimensional reductions which give non-Abelian theories in the two-dimensional spacetime and non-Abelian vortices in such theories.

Although the instantons in our setup are described by very simple BPS equations, the models we have considered have some exotic properties. For example, the action identically vanishes for any static configuration which is independent of one of the spatial coordinates. It is possible to modify the action to obtain a physically reasonable model without breaking the BPS properties and the Lifshitz scaling invariance. In particular, it is interesting to consider the anisotropic Skrymions in the Lifshitz-type model characterized by the superpotential which takes the form of the Wess-Zumino-Witten model.

The (2+1)-dimensional model we have considered in section 2.4 can be embedded into a supersymmetric model, in which the instanton configurations preserve a half of the supersymmetry. It should be very interesting to consider deformed supersymmetry algebras which allow us to compute the path integrals by means of the localization technique. For higher dimensions, the supersymmetry in the Lifshitz-type field theories has not yet been fully understood. We will discuss this topic in the near future.

\section{Acknowledgments}

This work is supported by the Japan Society for the Promotion of Science (JSPS) Grantin-Aid for Scientific Research (KAKENHI Grant No. 25400268) and by the Ministry of Education, Culture, Sports, Science, and Technology (MEXT)-Supported Program for the Strategic Research Foundation at Private Universities "Topological Science" (Grant No. S1511006). The work of M.N. is also supported in part by a Grant-in-Aid for Scientific Research on Innovative Areas "Topological Materials Science" (KAKENHI Grant No. 15H05855), "Nuclear Matter in Neutron Stars Investigated by Experiments and Astronomical Observations" (KAKENHI Grant No. 15H00841), and "Topological Quantum Phenomena" (KAKENHI Grant No. 25103720) from the MEXT of Japan. 


\section{A A supersymmetry in Lifshitz-type theories}

Let us consider the follwoing SUSY algebra in $(2+1)$-dimensional spacetime

$$
\{Q, \bar{Q}\}=2 i \partial_{t}, \quad Q^{2}=\bar{Q}^{2}=0,
$$

where $Q$ and $\bar{Q}$ are supercharges which transform as spinors under the spacial rotation in 2d Euclidean space

$$
Q \rightarrow e^{i \frac{\theta}{2}} Q, \quad \bar{Q} \rightarrow e^{-i \frac{\theta}{2}} \bar{Q} .
$$

Note that eq. (A.1) is a subalgebra in the standard $3 \mathrm{~d} \mathcal{N}=2$ SUSY algebra (4 real supercharges). The supercharges can be represented by the following derivatives in the superspace

$$
Q=\frac{\partial}{\partial \theta}+i \bar{\theta} \partial_{t}, \quad \bar{Q}=\frac{\partial}{\partial \bar{\theta}}+i \theta \partial_{t} .
$$

The supercharges anti-commute with

$$
D=\frac{\partial}{\partial \theta}-i \bar{\theta} \partial_{t}, \quad \bar{D}=\frac{\partial}{\partial \bar{\theta}}-i \theta \partial_{t} .
$$

The non-linear sigma model discussed in section 2.2 can be constructed in terms of the real multiplets

$$
\Phi^{a}=\phi^{a}+\theta \psi^{a}+\bar{\psi} \bar{\theta}^{a}+\theta \bar{\theta} F^{a}, \quad \bar{\Phi}=\Phi .
$$

The simplest action for the real multiplets is given by

$$
\mathcal{L}=-\int d \theta d \bar{\theta}\left[g_{a b}(\Phi) D \Phi^{a} \bar{D} \Phi^{b}+\mathcal{W}\left(\Phi, \partial_{i} \Phi, \cdots\right)\right],
$$

where $g_{a b}$ is the Riemann metric on the target space and $\mathcal{W}$ is a function of $\Phi, \partial_{i} \Phi$ and higher spatial derivatives. In terms of the component fields, the kinetic part takes the standard sigma model form

$$
\begin{aligned}
\mathcal{L}_{\text {kin }}= & g_{a b}\left[\partial_{t} \phi^{a} \partial_{t} \phi^{b}+\left(F^{a}+\Gamma_{c d}^{a} \psi^{c} \bar{\psi}^{d}\right)\left(F^{b}+\Gamma_{c d}^{b} \psi^{c} \bar{\psi}^{d}\right)+i\left(\psi^{a} \mathcal{D}_{t} \bar{\psi}^{b}-\mathcal{D}_{t} \psi^{a} \bar{\psi}^{b}\right)\right] \\
& -R_{a b c d} \psi^{a} \psi^{b} \bar{\psi}^{c} \bar{\psi}^{d} .
\end{aligned}
$$

The superpotential part is given by

$$
\mathcal{L}_{W}=\frac{\delta W}{\delta \phi^{a}} F^{a}+\mathcal{W}_{a b} \psi^{a} \bar{\psi}^{b}+\mathcal{W}_{a b}^{i}\left(\partial_{i} \psi^{a} \bar{\psi}^{b}+\psi^{b} \partial_{i} \bar{\psi}^{a}\right)+\mathcal{W}_{a b}^{i j}\left(\partial_{i} \psi^{a} \partial_{j} \bar{\psi}^{b}+\partial_{j} \psi^{b} \partial_{i} \bar{\psi}^{a}\right)+\cdots
$$

where we have defined

$$
\begin{aligned}
& \frac{\delta W}{\delta \phi^{a}}=\frac{\partial \mathcal{W}}{\partial \phi^{a}}-\partial_{i} \frac{\partial \mathcal{W}}{\partial\left(\partial_{i} \phi^{a}\right)}+\cdots \\
& \mathcal{W}_{a b}=\frac{\partial^{2} \mathcal{W}}{\partial \phi^{a} \partial \phi^{b}}, \quad \mathcal{W}_{a b}^{i}=\frac{\partial^{2} \mathcal{W}}{\partial\left(\partial_{i} \phi^{a}\right) \partial \phi^{b}}, \quad \mathcal{W}_{a b}^{i j}=\frac{\partial^{2} \mathcal{W}}{\partial\left(\partial_{i} \phi^{a}\right) \partial\left(\partial_{j} \phi^{b}\right)} .
\end{aligned}
$$

Eliminating the auxiliary fields $F^{a}$, we obtain the following bosonic part

$$
\mathcal{L}_{\text {bosonic }}=g_{a b} \partial_{t} \phi^{a} \partial_{t} \phi^{b}-g^{a b} \frac{\delta W}{\delta \phi^{a}} \frac{\delta W}{\delta \phi^{b}} .
$$

This becomes the Lagrangian discussed in section 2.2 after the Wick-rotation. The lower dimensional supersymmetric action can be obtained by the standard dimensional reduction along the spatial directions. 
Open Access. This article is distributed under the terms of the Creative Commons Attribution License (CC-BY 4.0), which permits any use, distribution and reproduction in any medium, provided the original author(s) and source are credited.

\section{References}

[1] E.M. Lifshitz, On the theory of second-order phase transitions I, Zh. Eksp. Teor. Fiz. 11 (1941) 255.

[2] E.M. Lifshitz, On the theory of second-order phase transitions II, Zh. Eksp. Teor. Fiz. 11 (1941) 269.

[3] P. Hořava, Membranes at quantum criticality, JHEP 03 (2009) 020 [arXiv:0812.4287] [INSPIRE].

[4] P. Hořava, Quantum gravity at a Lifshitz point, Phys. Rev. D 79 (2009) 084008 [arXiv:0901.3775] [INSPIRE].

[5] D. Anselmi and M. Halat, Renormalization of Lorentz violating theories, Phys. Rev. D 76 (2007) 125011 [arXiv:0707.2480] [INSPIRE].

[6] D. Anselmi, Weighted power counting and Lorentz violating gauge theories. I: General properties, Annals Phys. 324 (2009) 874 [arXiv:0808.3470] [INSPIRE].

[7] D. Anselmi, Weighted power counting and Lorentz violating gauge theories. II: Classification, Annals Phys. 324 (2009) 1058 [arXiv:0808.3474] [INSPIRE].

[8] T. Fujimori, T. Inami, K. Izumi and T. Kitamura, Power-counting and renormalizability in Lifshitz scalar theory, Phys. Rev. D 91 (2015) 125007 [arXiv: 1502.01820] [InSPIRE].

[9] D. Anselmi, Weighted scale invariant quantum field theories, JHEP 02 (2008) 051 [arXiv:0801.1216] [INSPIRE].

[10] S.R. Das and G. Murthy, $C P^{N-1}$ models at a Lifshitz point, Phys. Rev. D 80 (2009) 065006 [arXiv:0906.3261] [INSPIRE].

[11] K. Anagnostopoulos, K. Farakos, P. Pasipoularides and A. Tsapalis, Non-linear $\sigma$-model and asymptotic freedom at the Lifshitz point, arXiv:1007.0355 [INSPIRE].

[12] P.R.S. Gomes, P.F. Bienzobaz and M. Gomes, Competing interactions and the Lifshitz-type nonlinear $\sigma$-model, Phys. Rev. D 88 (2013) 025050 [arXiv: 1305.3792] [INSPIRE].

[13] P. Hořava, Quantum criticality and Yang-Mills gauge theory, Phys. Lett. B 694 (2010) 172 [arXiv: 0811.2217] [INSPIRE].

[14] B. Chen and Q.-G. Huang, Field theory at a Lifshitz point, Phys. Lett. B 683 (2010) 108 [arXiv:0904.4565] [INSPIRE].

[15] T. Kanazawa and A. Yamamoto, Asymptotically free lattice gauge theory in five dimensions, Phys. Rev. D 91 (2015) 074508 [arXiv:1411.4667] [inSPIRE].

[16] J. Alexandre, Lifshitz-type quantum field theories in particle physics, Int. J. Mod. Phys. A 26 (2011) 4523 [arXiv:1109.5629] [InSPIRE].

[17] H.J.W. Muller-Kirsten and D.H. Tchrakian, A Skyrme like lump in two Euclidean dimensions, J. Phys. A 23 (1990) L363 [inSPIRE]. 
[18] T. Gisiger and M.B. Paranjape, Solitons in a baby Skyrme model with invariance under volume/area preserving diffeomorphisms, Phys. Rev. D 55 (1997) 7731 [hep-ph/9606328] [INSPIRE].

[19] C. Adam, P. Klimas, J. Sanchez-Guillen and A. Wereszczynski, Compact baby Skyrmions, Phys. Rev. D 80 (2009) 105013 [arXiv:0909.2505] [INSPIRE].

[20] C. Adam, J. Sanchez-Guillen and A. Wereszczynski, A Skyrme-type proposal for baryonic matter, Phys. Lett. B 691 (2010) 105 [arXiv:1001.4544] [INSPIRE].

[21] C. Adam, T. Romanczukiewicz, J. Sanchez-Guillen and A. Wereszczynski, Investigation of restricted baby Skyrme models, Phys. Rev. D 81 (2010) 085007 [arXiv:1002.0851] [INSPIRE].

[22] J.M. Speight, Compactons and semi-compactons in the extreme baby Skyrme model, J. Phys. A 43 (2010) 405201 [arXiv:1006.3754] [InSPIRE].

[23] E. Bonenfant and L. Marleau, Nuclei as near BPS-Skyrmions, Phys. Rev. D 82 (2010) 054023 [arXiv: 1007.1396] [INSPIRE].

[24] C. Adam, J. Sanchez-Guillen and A. Wereszczynski, A BPS Skyrme model and baryons at large $N_{c}$, Phys. Rev. D 82 (2010) 085015 [arXiv:1007.1567] [InSPIRE].

[25] C. Adam, J. Sanchez-Guillen, A. Wereszczynski and W.J. Zakrzewski, Topological duality between vortices and planar Skyrmions in BPS theories with area-preserving diffeomorphism symmetries, Phys. Rev. D 87 (2013) 027703 [arXiv:1209.5403] [INSPIRE].

[26] C. Adam, C.D. Fosco, J.M. Queiruga, J. Sanchez-Guillen and A. Wereszczynski, Symmetries and exact solutions of the BPS Skyrme model, J. Phys. A 46 (2013) 135401 [arXiv:1210.7839] [INSPIRE].

[27] C. Adam, L.A. Ferreira, E. da Hora, A. Wereszczynski and W.J. Zakrzewski, Some aspects of self-duality and generalised BPS theories, JHEP 08 (2013) 062 [arXiv:1305.7239] [INSPIRE].

[28] C. Adam, J.M. Queiruga, J. Sanchez-Guillen and A. Wereszczynski, $N=1$ supersymmetric extension of the baby Skyrme model, Phys. Rev. D 84 (2011) 025008 [arXiv:1105.1168] [INSPIRE].

[29] C. Adam, J.M. Queiruga, J. Sanchez-Guillen and A. Wereszczynski, Extended supersymmetry and BPS solutions in baby Skyrme models, JHEP 05 (2013) 108 [arXiv:1304.0774] [INSPIRE].

[30] M. Nitta and S. Sasaki, BPS states in supersymmetric chiral models with higher derivative terms, Phys. Rev. D 90 (2014) 105001 [arXiv:1406.7647] [INSPIRE].

[31] M. Nitta and S. Sasaki, Classifying BPS states in supersymmetric gauge theories coupled to higher derivative chiral models, Phys. Rev. D 91 (2015) 125025 [arXiv:1504.08123] [INSPIRE].

[32] S. Bolognesi and W. Zakrzewski, Baby Skyrme model, near-BPS approximations and supersymmetric extensions, Phys. Rev. D 91 (2015) 045034 [arXiv: 1407.3140] [InSPIRE].

[33] C. Adam, T. Romanczukiewicz, J. Sanchez-Guillen and A. Wereszczynski, Magnetothermodynamics of BPS baby Skyrmions, JHEP 11 (2014) 095 [arXiv: 1405.5215] [INSPIRE].

[34] C. Adam, C. Naya, J. Sanchez-Guillen and A. Wereszczynski, Lifshitz field theories with SDiff symmetries, JHEP 03 (2013) 012 [arXiv: 1212.2741] [INSPIRE]. 
[35] A. Kobakhidze, J.E. Thompson and R.R. Volkas, BPS solitons in Lifshitz field theories, Phys. Rev. D 83 (2011) 025007 [arXiv: 1010.1068] [INSPIRE].

[36] I. Bakas, F. Bourliot, D. Lüst and M. Petropoulos, Geometric flows in Hor̆ava-Lifshitz gravity, JHEP 04 (2010) 131 [arXiv:1002.0062] [INSPIRE].

[37] I. Bakas, Gradient flows and instantons at a Lifshitz point, J. Phys. Conf. Ser. 283 (2011) 012004 [arXiv:1009.6173] [InSPIRE].

[38] E. Witten, Non-Abelian bosonization in two-dimensions, Commun. Math. Phys. 92 (1984) 455 [INSPIRE].

[39] G.H. Derrick, Comments on nonlinear wave equations as models for elementary particles, J. Math. Phys. 5 (1964) 1252 [inSPIRE].

[40] B.M.A.G. Piette, B.J. Schroers and W.J. Zakrzewski, Multisolitons in a two-dimensional Skyrme model, Z. Phys. C 65 (1995) 165 [hep-th/9406160] [InSPIRE].

[41] B.M.A.G. Piette, B.J. Schroers and W.J. Zakrzewski, Dynamics of baby Skyrmions, Nucl. Phys. B 439 (1995) 205 [hep-ph/9410256] [INSPIRE].

[42] I. Affleck, On constrained instantons, Nucl. Phys. B 191 (1981) 429 [inSPIRE].

[43] A.M. Polyakov and A.A. Belavin, Metastable states of two-dimensional isotropic ferromagnets, JETP Lett. 22 (1975) 245 [Pisma Zh. Eksp. Teor. Fiz. 22 (1975) 503] [INSPIRE].

[44] S.B. Gudnason and M. Nitta, Incarnations of Skyrmions, Phys. Rev. D 90 (2014) 085007 [arXiv:1407.7210] [INSPIRE].

[45] M. Kobayashi and M. Nitta, Winding Hopfions on $R^{2} \times S^{1}$, Nucl. Phys. B 876 (2013) 605 [arXiv: 1305.7417] [INSPIRE].

[46] M. Nitta, Fractional instantons and bions in the $O(N)$ model with twisted boundary conditions, JHEP 03 (2015) 108 [arXiv:1412.7681] [INSPIRE].

[47] M. Nitta, Fractional instantons and bions in the principal chiral model on $\mathbb{R}^{2} \times S^{1}$ with twisted boundary conditions, JHEP 08 (2015) 063 [arXiv: 1503.06336] [INSPIRE].

[48] E. Witten, Some exact multi-instanton solutions of classical Yang-Mills theory, Phys. Rev. Lett. 38 (1977) 121 [inSPIRE].

[49] P. Forgacs and N.S. Manton, Space-time symmetries in gauge theories, Commun. Math. Phys. 72 (1980) 15 [INSPIRE].

[50] B.J. Harrington and H.K. Shepard, Periodic Euclidean solutions and the finite temperature Yang-Mills gas, Phys. Rev. D 17 (1978) 2122 [inSPIRE].

[51] P. Rossi, Propagation functions in the field of a monopole, Nucl. Phys. B 149 (1979) 170 [INSPIRE].

[52] D.J. Gross, R.D. Pisarski and L.G. Yaffe, $Q C D$ and instantons at finite temperature, Rev. Mod. Phys. 53 (1981) 43 [INSPIRE].

[53] K.-M. Lee and P. Yi, Monopoles and instantons on partially compactified D-branes, Phys. Rev. D 56 (1997) 3711 [hep-th/9702107] [INSPIRE].

[54] K.-M. Lee, Instantons and magnetic monopoles on $R^{3} \times S^{1}$ with arbitrary simple gauge groups, Phys. Lett. B 426 (1998) 323 [hep-th/9802012] [INSPIRE]. 
[55] T.C. Kraan and P. van Baal, Exact T duality between calorons and Taub-NUT spaces, Phys. Lett. B 428 (1998) 268 [hep-th/9802049] [INSPIRE].

[56] T.C. Kraan and P. van Baal, Periodic instantons with nontrivial holonomy, Nucl. Phys. B 533 (1998) 627 [hep-th/9805168] [INSPIRE].

[57] T.C. Kraan and P. van Baal, Monopole constituents inside $\mathrm{SU}(N)$ calorons, Phys. Lett. B 435 (1998) 389 [hep-th/9806034] [INSPIRE].

[58] H. Kihara, Y. Hosotani and M. Nitta, Generalized monopoles in six-dimensional non-Abelian gauge theory, Phys. Rev. D 71 (2005) 041701 [hep-th/0408068] [INSPIRE].

[59] N.S. Manton and N. Sakai, Maximally non-Abelian vortices from self-dual Yang-Mills fields, Phys. Lett. B 687 (2010) 395 [arXiv: 1001.5236] [INSPIRE].

[60] M. Eto, T. Fujimori, M. Nitta and K. Ohashi, All exact solutions of non-Abelian vortices from Yang-Mills instantons, JHEP 07 (2013) 034 [arXiv:1207.5143] [INSPIRE]. 